läßt daher die Periodizität des Ansatzes (5) unverändert; damit bestimmen sich die Einelektronen-Wellenfunktionen $u$ aus einer Fockschen Gleichung, in welcher das lineare Potential des Feldes durch das SägezahnPotential ersetzt ist. Man gelangt nicht zu der W Annierschen Gl. (4), hat vielmehr an Stelle des dortigen Feldgliedes das periodische Potential $V(r)$ durch das Sägezahn-Potential zu ergänzen. Die besten EinelektronenWellenfunktionen (2) sind somit nicht von der W schen Gestalt, sondern gleichen im wesentlichen den von
Houston benützten; die Zenerschen Übergänge finden statt, die Übergangswahrscheinlichkeiten berechnen sich nach den Formeln von Houstos mit einer unbedeutenden Modifikation. Es bleibt hinzuzufügen, daß der Beitrag des Sägezahn-Potentials zur Gesamtenergie davon abhängt, wo im Raum die Zellengrenzen gelegt werden. Sieht man alle möglichen Lagen der Zellengrenzen als gleichberechtigt an und mittelt über sie, so verschwindet der Beitrag des Sägezahn-Potentials zur Gesamtenergie und man kehrt genau zur Houstonschen Theorie zurück.

\section{Über die Relaxationszeiten und Eigenfunktionen des Maxwellschen Gases}

\section{Von L. WaLdManN}

Max-Planck-Institut für Chemie, Mainz

(Z. Naturforschg. 11 a, 523—524 [1956] ; eingegangen am 8. Mai 1956)

Es werde ein reines, räumlich homogenes Gas betrachtet, dessen Zustand wenig von dem durch die Verteilungsfunktion

$$
f^{(\mathbf{0})}=n_{0}(\beta / \pi)^{3 / 2} e^{-\beta c^{2}}, \quad \beta=m / 2 k T_{0}
$$

beschriebenen, thermischen Gleichgewichtszustand abweicht (c Molekulargeschwindigkeit, $m$ Molekülmasse, $n_{0}$ Teilchenkonzentration, $T_{0}$ Temperatur). Für die Verteilungsfunktion des Gases werde daher gesetzt

$$
f(t, \mathfrak{c})=f^{(\mathbf{0})}[1+\Phi(t, \mathfrak{c})],
$$

wo dann im wesentlichen $\Phi \ll 1$. Für $\Phi$ gilt die genäherte (linearisierte) Boltzmann-Gleichung

$$
f^{(0)} \frac{\partial \Phi}{\partial t}=-n_{0}^{2} I(\Phi) \text {. }
$$

Der linearisierte Stoßoperator $I$ ist bekanntlich ${ }^{1}$ durch $n_{0}{ }^{2} I(\Phi)=\iiint f^{(0)} f_{1}{ }^{(0)}\left(\Phi+\Phi_{1}-\Phi^{\prime}-\Phi_{1}{ }^{\prime}\right) g b \mathrm{~d} b \mathrm{~d} \varepsilon \mathrm{dc}_{1}$ gegeben $\left(\Phi_{1}=\Phi\left(\mathfrak{c}_{1}\right)\right.$ usw., $\mathfrak{c}^{\prime}, \mathfrak{c}_{1}{ }^{\prime}$ Geschwindigkeiten nach dem Stoß, $g=\left|\mathfrak{c}-\mathfrak{c}_{1}\right|, b=$ Stoßparameter, $\varepsilon=$ Azimutwinkel von $\mathfrak{g}^{\prime}$ um $\mathfrak{g}, \mathrm{dc}_{1}=\mathrm{d} c_{1 x} \mathrm{~d} c_{1 y} \mathrm{~d} c_{1 z}$ ).

Nun sei die spezielle Zeitabhängigkeit

$$
\Phi(t, \mathrm{c})=e^{-\omega t} \psi(\mathrm{c})
$$

angenommen. Für $\psi(\mathfrak{c})$ gilt dann nach (1)

$$
n_{0}^{2} I(\psi)=\omega f^{(\mathbf{0})} \psi \text {. }
$$

Das ist eine Eigenwertgleichung, denn $\psi$ darf für $c \rightarrow \infty$ nicht zu stark anwachsen, damit $f^{(0)}(1+\psi)$ eine physikalisch sinnvolle Verteilungsfunktion darstellt. Die Eigenwerte $\omega$ sind die reziproken Relaxationszeiten des Gases. Für zwei Eigenfunktionen $\psi_{\mathrm{N}}, \psi_{\mathrm{N}^{\prime}}$ zu verschiedenen Eigenwerten beweist man leicht die Orthogoralitätsrelation

$$
\int \psi_{\mathrm{N}} \psi_{\mathrm{N}^{\prime}} f^{(\mathbf{0})} \mathrm{d} \mathrm{c}=0 .
$$

Für Maxwellsche Moleküle, d. s. punktförmige Zentren, die sich mit der Kraft proportional $r^{-5}$ abstoßen ( $r=$ Abstand), kann man sämtliche Eigenfunktionen

1 s. z. B. S. Chapman u. T. G. Cowling, Mathematical Theory of Non-Uniform Gases, Cambridge 1939, S. 110. und Eigenwerte explizit angeben. Der folgende Weg führt dazu. Es seien die Funktionen

$$
\Phi_{l}\left(\beta c^{2}, s\right)=(1-s)^{-3 / 2-l} e^{-\beta c^{2} s /(1-s)}
$$

betrachtet. Es läßt sich zeigen, daß

$$
\begin{aligned}
& n_{0} I\left[\Phi_{0}\left(\beta c^{2}, s\right)\right] \\
& =f^{(0)} \int_{0}^{\infty}\left[\Phi_{0}\left(\beta c^{2}, s\right)\right. \\
& \left.\quad+1-\Phi_{0}\left(\beta c^{2}, s^{\prime}\right)-\Phi_{0}\left(\beta c^{2}, s_{1}{ }^{\prime}\right)\right] 2 \pi g b \mathrm{~d} b, \\
& \text { wo } s^{\prime}=s \sin ^{2} \Theta, s_{1}{ }^{\prime}=s \cos ^{2} \Theta
\end{aligned}
$$$$
\text { und } \quad \Theta=\pi / 2-\chi / 2, \quad \chi=\Varangle\left(\mathfrak{g} \mathfrak{g}^{\prime}\right) \text {. }
$$

Nun ist bekanntlich $\Phi_{l}$ die erzeugende Funktion der Sonineschen Polynome $S_{l_{+2}}^{(r)}$; es gilt ${ }^{2}$

$$
S_{l+\frac{1}{2}}^{(r)}(x)=\left.\frac{1}{r !}\left(\frac{\partial}{\partial s}\right)^{r} \Phi_{l}(x, s)\right|_{s \rightarrow 0} .
$$

Somit entnimmt man aus (3), indem man beiderseits die Differentiation $\left.(\partial / \partial s)^{r}\right|_{s \rightarrow 0 \text { ausführt, }}$

$$
n_{0}^{2} I\left[S_{l+\frac{1}{2}}^{(r)}\left(\beta c^{2}\right)\right]=\omega_{0 r} f^{(0)} S_{12}^{(r)}\left(\beta c^{2}\right),
$$

wobei $\omega_{00}=0$,

$$
\begin{array}{r}
\omega_{0 r}=n_{0} 2 \pi \int_{0}^{\infty}\left(1-\sin ^{2} r \Theta-\cos ^{2} r \Theta\right) g b \mathrm{~d} b, \\
r>0 .
\end{array}
$$

Damit hat man alle kugelsymmetrischen Eigenfunktionen im Sinn von (2) des Maxwellschen Gases und die zugehörigen Eigenwerte. - Um die nichtkugelsymmetrischen Eigenfunktionen zu finden, berechnet man zunächst $I\left[e^{-\beta(\mathfrak{c}-\mathfrak{v})^{2} \cdot s /(\mathfrak{c}-\mathfrak{v})}\right]$, wo $\mathfrak{v}$ eine beliebige feste Geschwindigkeit ist, entwickelt dann nach Kugelfunktionen und läßt schließlich $\mathfrak{v} \rightarrow 0$ gehen. Man erhält so als Verallgemeinerung von (3)

$$
\begin{aligned}
n_{0}{ }^{2} I\left[S_{l+\frac{1}{2}}^{(r)}\left(\beta c^{2}\right) c^{l} Y_{l m}(\vartheta, \varphi)\right] \\
=\omega_{l r} f^{(0)} S_{l+\frac{1}{2}}^{(r)}\left(\beta c^{2}\right) c^{l} Y_{l m}(\vartheta, \varphi),
\end{aligned}
$$

worin $\vartheta, \varphi$ Polarwinkel von c und $Y_{l m}$ die Kugelflächenfunktionen sind. Für die Eigenwerte gilt

$$
\omega_{l r}=n_{0} \cdot 2 \pi \int_{0}^{\infty}\left[1-\sin ^{l+2 r} \Theta P_{l}(\sin \Theta) \quad(7)\right.
$$

( $P_{l}=$ Legendresches Polynom). Wieder begegnet man also den von Burnetr ${ }^{3}$ in die Gastheorie eingeführten

2 vgl. Chapman u. Cowling, l. c. ${ }^{1}$, S. 123.

3 D. Burnett, Proc. London Math. Soc. 39, 385 [1935]. 
Sonineschen Polynomen. Diese hängen wie geschildert aufs engste zusammen mit den Eigenfunktionen des Maxwellschen Gases.

Aus (7) erkennt man, daß $\omega_{l r} \rightarrow \infty$ mit $l+2 r \rightarrow \infty$, d. h. die Eigenwerte häufen sich nur bei $\omega=\infty$, das Spektrum ist diskret. Für die Eigenfunktionen zu verschiedenen $l$-Werten gelten übrigens die Formeln

$$
\begin{aligned}
& \omega_{1 r}=\omega_{0, r+1} \\
& \omega_{2 r}=\frac{3}{2} \omega_{0, r+2}-\frac{1}{2} \omega_{0, r+1} \\
& \omega_{3 r}=\frac{5}{2} \omega_{0, r+3}-\frac{3}{2} \omega_{0, r+2} \quad \text { usw. }
\end{aligned}
$$

Die Zahlenkoeffizienten sind die Koeffizienten des entsprechenden Legendreschen Polynoms.

Einige Relaxationszeiten wurden von $\mathrm{M}_{\mathrm{AXwELL}}{ }^{4}$ selbst berechnet. Bei Chapman und Cowling werden die Eigenfunktionen zu $(l, r)=(1,1)$ und $(2,0)$ angegeben und verifiziert (l. c. ${ }^{1}$, S. $\left.175-177\right)$. Die allgemeinen Formeln (6) und (7) scheinen bisher in der Literatur nicht vorzukommen *.

4 J. C. Maxwell (1879), Scient. Papers 2, S. 694-696; s. a. L. Boltzmann, Vorlesungen über Gastheorie, I. Teil, Leipzig 1895, S. $164-176$.

* A n m. b. d. K o r r. : Aus der Arbeit von Ikenberry und Truesdell, J. Rat. Mech. Analysis 5, l [1956] entnahm ich inzwischen, daß die obigen Eigenwerte offenbar schon von $W_{\text {ang }}$ Chang und Uhlenbeck in dem hier nicht verfügbaren Bericht des Univ. Michig. Eng. Res. Inst. Project M 999, Oktober 1952 aufgestellt worden sind.

\section{BESPRECHUNG}

Die wissenschaftliche und angewandte Photographie. 1. Band: Das photographische Objektiv. Von Jo h a n n e s F l üg g e. Springer-Verlag, Wien 1955. XIII, 373 S. mit 196 Abb.; Preis geb. DM 69.-.

Das seit einigen Jahren vergriffene $\mathrm{H}_{\mathrm{AY}}$-v. RoHrsche Handbuch der wissenschaftlichen und angewandten Photographie wird jetzt in Form einer Folge von selbständigen, einzeln käuflichen Monographien von $\mathrm{K}$. Michel neu herausgegeben. Der vorliegende erste Band von J. F LüGGE verfolgt zwei Ziele: Die ersten 7 Kapitel geben auf 150 Seiten eine klare und auch für die praktische Anwendung gut brauchbare Übersicht über die Grundlagen der geometrischen Optik und die Theorie der Abbildungsfehler, dabei wird auch der Standpunkt der Wellenoptik berücksichtigt. Der zweite Teil bringt eine systematische Darstellung der verschiedenen Aus- führungsformen photographischer Objektive; er wird ergänzt durch die Bildfehleranalysen und Konstruktionsdaten von 36 typischen Objektiven, durch die in einer Folge von Abbildungen wiedergegebenen Ergebnisse der Strahlendurchrechnung eines Beispiels (Xenon $1: 2,0)$ und durch eine ziemlich vollständige Tabelle der Markenobjektive und ihrer Eigenschaften. Kapitel über die optischen Werkstoffe, über Lichtfilter und Polarisatoren, über Prüf- und Meßverfahren und ein von L. Bierwagen beigesteuerter Abschnitt über Entspiegelung runden die für den Konstrukteur wie den Benutzer photogaphischer Objektive gleich wertvolle Monographie ab, der der Verlag eine ausgezeichnete Ausstattung gegeben hat.

H. Siedentopf, Tübingen. 